\title{
Actividad nematicida de extractos foliares de cuatro especies de Piper contra Caenorhabditis elegans
}

\section{Nematicidal activity of four Piper species foliar extracts against Caenorhabditis elegans}

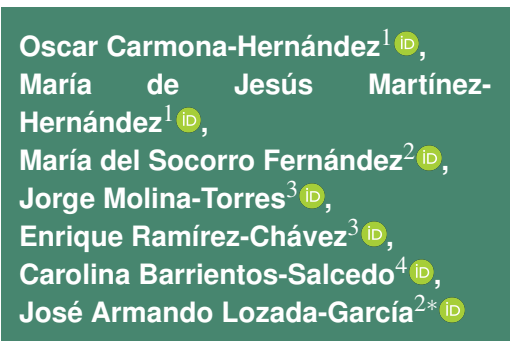

${ }^{1}$ Facultad de Ciencias Agrícolas, Universidad Veracruzana. Circuito Universitario Gonzalo Aguirre Beltrán $\mathrm{s} / \mathrm{n}$, Col. Zona Universitaria, CP. 91090. Xalapa, Veracruz, México.

${ }^{2}$ Facultad de Biología Xalapa, Universidad Veracruzana. Circuito Universitario Gonzalo Aguirre Beltrán $\mathrm{s} / \mathrm{n}$, Col. Zona Universitaria, CP. 91090. Xalapa, Veracruz, México.

${ }^{3}$ Departamento de Biotecnología y Bioquímica CINVESTAV-IPN Unidad Irapuato, Libramiento Norte Km 9.5. CP. $36500 . \quad$ Irapuato, Guanajuato, México.

${ }^{4}$ Facultad de Bioanálisis Veracruz, Universidad Veracruzana, Iturbide $\mathrm{s} / \mathrm{n}$ Col. Centro, CP. 91700. Veracruz, Veracruz, México.

*Autor de correspondencia: alozada@uv.mx

Artículo científico

Recibido: 03 de marzo 2021 Aceptado: 29 de octubre 2021

Como citar: Carmona-Hernández $\mathrm{O}$ Martínez-Hernández MJ, Fernández MS, Molina-Torres J, Ramírez-Chávez E, Barrientos-Salcedo C, LozadaGarcía JA (2021) Actividad nematicida de extractos foliares de cuatro especies de Piper contra Caenorhabditis elegans. Ecosistemas y Recursos Agropecuarios 8(3): e2943. DOI: 10.19136/era.a8n3.2943
RESUMEN. Las especies del género Piper han mostrado potencial como biocidas naturales en diferentes organismos, lo cual es atribuible a la diversidad de metabolitos secundarios que sintetizan. Por lo cual, el objetivo de esta investigación fue evaluar el efecto nematicida contra Caenorhabditis elegans de los extractos hidroacohólicos, etanólicos y metanólicos de cuatro especies del género Piper. Los resultados mostraron que el extracto de $P$. aduncum fue el que presentó mayor actividad con una concentración letal media $\left(\mathrm{CL}_{50}\right)$ estimada en $0.650 \mathrm{mg} \mathrm{mL}^{-1}$. Se determinaron perfiles cromatográficos por HPTLC y en GC-EIMS de los extractos de mayor actividad. Los metabolitos identificados fueron alcaloides, flavonoides, terpenos, esteroles, cumarinas y saponinas. En la GC-EIMS se identificaron 67 compuestos potenciales en los extractos etanólicos. Se concluye que el extracto etanólico de Piper aduncum presentó mayor actividad nematicida $\left(\mathrm{CL}_{50}\right.$ de $0.650 \mathrm{mg} \mathrm{mL}^{-1}$ ) y el de mayor número de compuestos identificados preliminarmente.

Palabras clave: Bioactividad, CG-EIMS, $\mathrm{CL}_{50}$, HPTLC, Probit.

ABSTRACT. Piper spices haven showed a great biological potential as natural biocide, in different organism. This is attributed to the diversity bioactive metabolites present in tissue. In this research we evaluated the nematicidal activity of different foliar extracts from Piper spices against Caenorhabditis elegans. We evaluated the nematicide activity of the hydroalcoholic, methanolic and ethanolic extracts. Chromatography prolife by HTPLC and GC-EIMS of the extracts with mayor activity was obtained. The ethanolic extract from Piper aduncum displayed higher activity as lethal concentration $50\left(\mathrm{LC}_{50}\right)$ estimated at $0.650 \mathrm{mg} \mathrm{mL}^{-1}$. The bioactive metabolites identified were alkaloids, flavonoids, terpenoids, steroids and coumarins. The GC-EIMS determinate 67 compounds in the higher activity extracts. Concluded the $P$. aduncum ethanolic extract has the higher activity and the mayor compounds diversity.

Key words: Bioactivity, CG-EIMS, HPTLC, LC 50 , Probit. 


\section{INTRODUCCIÓN}

El género Piper comprende cerca de 1500 especies distribuidas en las zonas tropicales, siendo más abundantes en bosques húmedos premontanos y tierras bajas, de éstas, 136 se encuentran en México y 89 en Veracruz (Jaramillo et al. 2008, Villaseñor 2016). Las especies a este género representan un recurso biotecnológico importante en la búsqueda de compuestos bioactivos. Al respecto, se ha reportado que poseen actividad bactericida, insecticida, antiprotozoaria, fungicida, antihelmíntica y nematicida, atribuibles a sus más de $700 \mathrm{com}$ puestos fitoquímicos identificados (Scott et al. 2008, Carmona-Hernández et al. 2016).

Los nemátodos fitopatógenos cusan daño anualmente en aproximadamente un $7 \%$ de la producción agrícola mundial, lo cual equivale a 48991.04 toneladas y en un estimado de 215769.92 millones de dólares (Abd-Elgawad y Askary 2015). Algunos fitonemátodos responsables de los daños pertenecen a los géneros Melodoigyne y Globodera (Pehlivan et al. 2020), y son causantes de malformaciones en plantas de la familia Solanaceae, así como marchitamiento en algunas hortalizas y plantaciones de café (Del-Prado-Vera et al. 2018). Aunado a esto, muchas especies de estos géneros han mostrado resistencia o tolerancia a los nematicidas sintéticos (Roeber et al. 2013, Wram y Zasada 2020). Como alternativa al uso y aplicación de estos compuestos, se ha sugerido el uso de extractos naturales de plantas, en particular de las especies de Piper que se han evaluado como plaguicidas alternativos por su capacidad toxicológica. Por lo que el objetivo del presente trabajo fue determinar el perfil fitoquímico y efecto nematicida de los extractos foliares de cuatro especies de Piper contra Caenorhabditis elegans, nemátodo ampliamente utilizado como modelo en bioensayos de actividad biológica por su amplia capacidad de resistencia toxicológica frente a extractos naturales y su estrecha relación filogenética con nemátodos fitopatógenos (Wang et al. 2012).

\section{MATERIALES Y MÉTODOS}

\section{Colecta e identificación de especímenes de Piper}

Las especies utilizadas fueron: Piper amalago

L., Piper aduncum L., Piper marginatum Jacq., y Piper umbellatum L., las cuales se colectaron en los municipios de Coatepec (19.436502, -96.955859) y San Andrés Tlalnelhuayocan (19.516908, 96976465), en el estado de Veracruz, México. La identificación se realizó en el Herbario XAL del Instituto de Ecología, y con la base de acceso abierto de la Universidad Nacional Autónoma de México (UNAM), Intermountain Regional Herbarium Network y Botanischer Garten und Botanisches Museum Berlin-Dahlem (Carmona-Hernández et al. 2014).

\section{Preparación de extractos}

Aproximadamente $1.5 \mathrm{~kg}$ de hojas de cada especie se secaron a $50 \pm 5{ }^{\circ} \mathrm{C}$ en una estufa, para posteriormente obtener un polvo fino (Soberon et al. 2006). A partir de este, se realizaron extracciones a reflujo continuo con etanol (EtOH), metanol (MetOH) y etanol-agua 50:50 ( $\left.\mathrm{EtOH}: \mathrm{H}_{2} \mathrm{O}\right)$, en un equipo Soxhlet por $10 \mathrm{~h}$ continuas. Los extractos obtenidos se concentraron a presión reducida en un rotavapor a $50^{\circ} \mathrm{C}$ y se estimó el rendimiento bruto (Moreno et al. 2000, Wiratno et al. 2009, Agyare et al. 2014).

\section{Cultivo de Caenorhabditis elegans}

La cepa N2 de $C$. elegans fue obtenida del laboratorio Evolution of Metabolic Diversity (LANGEBIO) y se cultivó a temperatura promedio de $22 \pm$ $2{ }^{\circ} \mathrm{C}$, en cajas Petri con nematode medium growth (NMG) (17 g de agar bacteriológico, $2.5 \mathrm{~g}$ de peptona, $3 \mathrm{~g}$ de $\mathrm{NaCl}, 1 \mathrm{~mL}$ de $\mathrm{CaCl}_{2} 1 \mathrm{M}, 1 \mathrm{~mL}$ de $\mathrm{MgSO}_{4} 1 \mathrm{M}, 25 \mathrm{~mL}$ de $\mathrm{KH}_{2} \mathrm{PO}_{4} 1 \mathrm{~mL}$ de solución de colesterol de $5 \mathrm{mg} \mathrm{mL}^{-1}$ en $\mathrm{EtOH}$ ) e inoculadas previamente con Escherichia coli cepa OP50, la cual sirvió de alimento para los nemátodos (Katiki et al. 2011, Sant'anna et al. 2013).

\section{Bioensayos de toxicidad}

Se usaron nemátodos en instar larvar L4, para ello se sincronizaron por el método de blancamiento alcalino, los gusanos se extrajeron de las cajas Petri 
con NMG y con solución Buffer M9 $\left(3 \mathrm{~g} \mathrm{KH}_{2} \mathrm{PO}_{4}, 6 \mathrm{~g}\right.$ $\mathrm{Na}_{2} \mathrm{HPO}_{4}, 5 \mathrm{~g} \mathrm{NaCl}, 0.25 \mathrm{~g} \mathrm{MgSO}_{4} 7 \mathrm{H}_{2} \mathrm{O}$ en un litro de agua) (Porta de la Riva et al. 2012, Agyare et al. 2014, Ow y Hall 2015).

Se realizaron los ensayos en microplacas de 96 pozos, en cada microcelda se depositaron 10 larvas $L 4$, con diferentes concentraciones de los extractos aleatorizados más un control (Buffer M9). El diseño experimental consistió en cinco concentraciones equidistantes del extracto de cada especie disuelto en Buffer M9, con cinco repeticiones, cada una de ellas correspondía a una placa. La exposición fue por $24 \mathrm{~h}$ a temperatura $22 \pm 2{ }^{\circ} \mathrm{C}$, transcurrido el tiempo se contabilizó el número de organismos sobrevivientes, mediante la inspección visual con ayuda de un microscopio estereoscópico (Roh y Choi 2007, Katiki et al. 2011, Agyare et al. 2014).

\section{Análisis estadístico}

El diseño experimental utilizado fue completamente al azar para cada extracto y se compararon entre las diferentes concentraciones, para determinar diferencias significativas y poder estimar una curva dosis-repuesta. Los resultados se expresaron en medias, graficadas en el programa Statistica 10. Se realizó un análisis de varianza (ANOVA) de una vía, y para todos los casos se utilizó la prueba de Tukey para la comparación de medias. El cálculo de la $\mathrm{CL}_{50}$ se estimó mediante el modelo de regresión lineal Probit, donde la concentración de cada extracto fue la variable independiente y la mortalidad de las larvas en porcentaje, la variable dependiente (Finney 1987, Jensen et al. 2006), para lo cual se utilizó el programa estadístico BioStat V5.

\section{Caracterización fitoquímica preliminar y perfiles por HTPLC}

Los metabolitos secundarios se identificaron por pruebas fitoquímicas preliminares, para alcaloides, flavonoides, saponinas, cumarinas y triterpenos y esteroles, de acuerdo con una escala cualitativa (Domínguez 1979, Carmona-Hernández et al. 2014). Además, se realizaron perfiles químicos para alcaloides, terpenos, compuestos fenólicos, cumarinas y saponinas usando el equipo High Per- fomance Thin Layer Chromatography (HTPLC) (CAMAG Mutenz, Switzerland) (Lebot et al. 2014, Riffault et al. 2014). Las placas desarrolladas se digitalizaron con el software Wincats 1.4.3 y fueron tomadas con el equipo TLC Visualizar (CAMAG) a longitudes de onda de 360 y $260 \mathrm{~nm}$, luz blanca visible superior e inferior. Con la ayuda del software Wincats 1.4 .3 se estimaron los factores de retención (Rf) y se realizaron los perfiles gráficos mediante el modelado de bandas (Annegowda et al. 2013, Lebot et al. 2014, Upadhya et al. 2016).

\section{Análisis por Cromatografía de Gases asociada a Espectrometría de Masas con ionización por Im- pacto Electrónico (CG-EIMS)}

El análisis se realizó para los extractos de mayor actividad usando un equipo de GC-EIMS Agilent Technologies serie 7890A, acoplado a un detector selectivo de masas con ionización por impacto electrónico y separación por cuadrupolo de la marca Agilent Technologies serie 5979C. La separación de los compuestos se realizó en una columna DB1MS UI Agilent Technologies $(60 \mathrm{~m} \times 250 \mathrm{~mm} \times 0.25 \mathrm{~mm})$, se utilizó Helio de ultra alta pureza (99.9999) como gas acarreador a un flujo de $1 \mathrm{~mL} \mathrm{~min}^{-1}$; la temperatura de inyección fue de $250{ }^{\circ} \mathrm{C}$. La programación de la corrida fue: temperatura inicial $150{ }^{\circ} \mathrm{C}$ durante 3 min, $4{ }^{\circ} \mathrm{C} \mathrm{min}{ }^{-1}$ a $300{ }^{\circ} \mathrm{C}$ por $13 \mathrm{~min}$. Los compuestos fueron identificados mediante la base de datos NIST 11 (National Institute Standard and Tecnology) (Robles-Zepeda et al. 2006).

\section{RESULTADOS}

\section{Actividad nematicida en $C$. elegans}

En 11 de los 12 extractos evaluados se determinó actividad nematicida sobre $C$. elegans. Los extractos etanólicos fueron los de mayor actividad, seguidos de los hidroalcohólicos y los metanólicos. Los extractos etanólicos de las cuatro especies tuvieron mortalidades superiores al $90 \%$. En los hidroalchólicos la actividad fue variada, mientras que para $P$. umbellatum la mortalidad fue del $100 \%$ a 3 $\mathrm{mg} \mathrm{mL}^{-1}$, para $P$. amalago no superó el $40 \%$ de mortalidad. De los extractos metanólicos, el menos ac- 
tivo fue el de $P$. marginatum con $0 \%$ de mortalidad, en contraste el extracto de $P$. amalago presentó una mortalidad mayor a $90 \%$ seguido de P. umbellatum (Figura 1).

La $\mathrm{CL}_{50}$ estimada para el extracto etanólico de $P$. aduncum fue de $0.650 \mathrm{mg} \mathrm{mL}^{-1}$, siendo la de mayor toxicidad con menor concentración. Seguida del extracto etanólico de $P$. marginatum con 0.836 $\mathrm{mg} \mathrm{mL}^{-1}$, P. umbellatum con una $\mathrm{CL}_{50}$ de 1.389 $\mathrm{mg} \mathrm{mL}^{-1}$ y finalmente $P$. amalago tuvo una $\mathrm{CL}_{50}$ de $2.962 \mathrm{mg} \mathrm{mL}^{-1}$ (Tabla 1). Los extractos hidroalcohólicos presentaron actividad biológica en rangos no superiores al $80 \%$; el de $P$. marginatum tuvo una $\mathrm{CL}_{50}$ en $2.214 \mathrm{mg} \mathrm{mL}^{-1}$, seguida de $P$. umbellatum con $3.101 \mathrm{mg} \mathrm{mL}^{-1}$ y $P$. amalago con una $\mathrm{CL}_{50}$ de $10.907 \mathrm{mg} \mathrm{mL}^{-1}$. Para $P$. aduncum la concentración letal media estimada fue de $58.677 \mathrm{mg} \mathrm{mL}^{-1}$, siendo el menos activo sobre $C$. elegans. Los extractos metanólicos fueron los de menor actividad comparados con los etanólicos y los hidroalcohólicos, donde las $\mathrm{CL}_{50}$ para $P$. aduncum fue de $168.741 \mathrm{mg} \mathrm{mL}^{-1}$, $P$. umbellatum de $4.630 \mathrm{mg} \mathrm{mL}^{-1}$, P. amalago de $4.161 \mathrm{mg} \mathrm{mL}^{-1}$ y $P$. marginatum no tuvo actividad en C. elegans.

\section{Análisis fitoquímicos y perfiles por HTPLC}

Mediante las pruebas fitoquímicas preliminares se identificó la presencia de alcaloides y flavonoides en los 12 extractos, las cumarinas estuvieron ausentes en los extractos de P. umbellatum, los terpenos y esteroles no se detectaron en los extractos etanólicos, y las saponinas solo se identificaron en los extractos hidroalcohólicos (Tabla 2). Por HTPLC se identificaron las las bandas correspondientes a $\mathrm{Rf}=0.5$ y 0.6 pertenecientes a alcaloides en los tres extractos de $P$. amalago. Los extractos de P. marginatum tuvieron presencia de alcaloides, con Rf similar en las bandas 0.5 y 0.6 ; y en Piper umbellatum fue la banda de $\mathrm{Rf}=0.6$ (Tabla 3 ).

Los flavonoides estuvieron ausentes en el extracto hidroalcohólico de $P$. umbellatum, en cambio para metanol y etanol se encontró una sola banda cada una con $\mathrm{Rf}=0.739$ y 0.900 , respectivamente. Los flavonoides en $P$. marginatum se presentaron en los tres extractos. En P. amalago, el extracto etanólico presentó el mayor número de bandas con $\mathrm{Rf}=0.752$, $0.876,0.885$. Finalmente, para los extractos de $P$. aduncum se determinó un Rf similar a 0.800 .

Las cumarinas estuvieron presentes en todos los extractos, y el hidroalcohólico de $P$. amalago tuvo el mayor número de bandas con siete. Las saponinas fueron los compuestos de mayor abundancia después de los terpenos. El extracto etanólico de $P$. aduncum tuvo 14 bandas con $\mathrm{Rf}=0.057,0.17$, $0.182,0.287,0.329,0.44,0.512,0.5140 .681,0.746$, $0.788,0.795,0.863,0.969$. Los terpenos estuvieron presentes en todas las muestras analizadas, estando mayormente representados en el extracto etanólico de $P$. marginatum con 12 bandas con $\mathrm{Rf}=0.025$, $0.096,0.106,0.179,0.241,0.267,0.292,0.429$, 0.485, 0.559, 0.697, 0.774 (Tabla 3).

\section{Análisis por GC-EIMS}

En la caracterización química tentativa de los extractos etanólicos por cromatografía de gases acoplada a espectrometría de masas (GC-EIMS), se registró en su mayoría compuestos de origen terpénico y aceites esenciales, así como algunas cumarinas y compuestos fenólicos. Otros compuestos identificados tentativamente fueron cadaleno, $\delta$ cadineno, $\beta$ copaeno, $\alpha$-gurjuneno y calameneno para $P$. umbellatum (Tabla 4).

En $P$. aduncum se identificaron germacreno $D$, ácido (Z)-cinámico, $\gamma$ sitosterol, estigmasterol, ácido mirístico y humuleno (Tabla 5); en $P$. marginatum: cadaleno, $\alpha$ calacoreno, aromandendreno, ácido cinámico, cis-muurol-3,5-dieno (Tabla 6); y para P. amalago: piranona, coumarano, piperonal y 2nonadecano (Tabla 7). Finalmente, para todos los extractos analizados se identificaron tentativamente ácido esteárico, ácido linoleico y ácido palmítico.

\section{DISCUSIÓN}

La mayoría de los extractos de las especies de Piper mostraron actividad nematicida contra C. elegans a bajas concentraciones, lo que coincide con lo reportado para otras especies del mismo género (Atjanasuppat et al. 2009). Los extractos etanólicos de las cuatro especies mostraron ser más ac- 


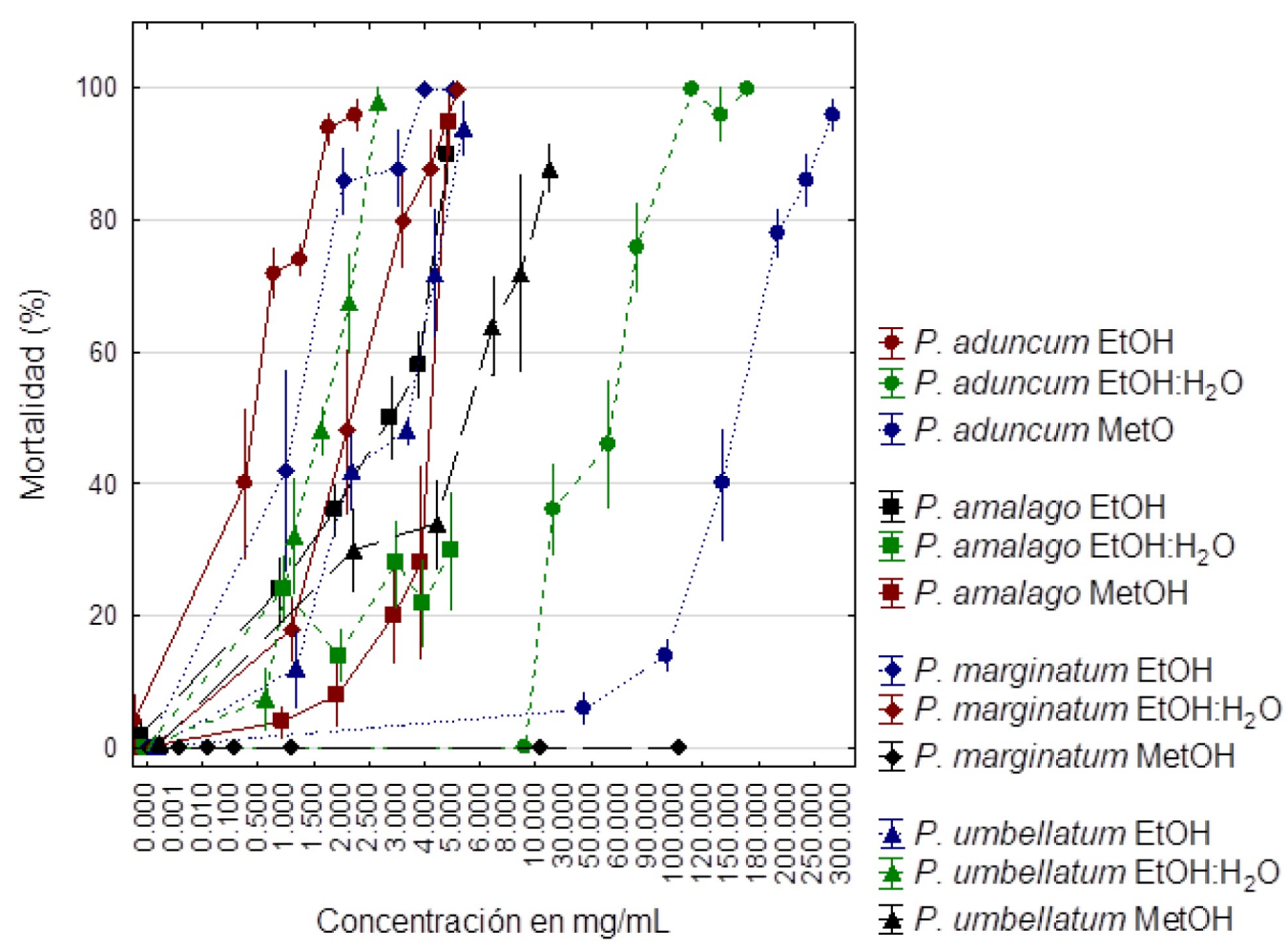

Figura 1. Mortalidad de Caenorhabditis elegans, después de $24 \mathrm{~h}$ de exposición a extractos de Piper spp. $\mathrm{P}<0.05$, a excepción del extracto metanólico de P. marginatum $\mathrm{p}>0.05 \mathrm{EtOH}-\mathrm{Etanol}$, MetOH-Metanol, EtOH: $\mathrm{H}_{2} \mathrm{O}$ : Hidroalcohólico al $70 \%$.

Tabla 1. Concentración letal media $\left(\mathrm{CL}_{50}\right)$ de los extractos de las especies de Piper en C. elegans.

\begin{tabular}{|c|c|c|c|}
\hline & Extracto & $\begin{array}{l}\text { Concentración Letal Media }\left(\mathrm{CL}_{50}\right) \\
\qquad \mathrm{mg} \mathrm{mL}^{-1}\end{array}$ & Error estándar \\
\hline & $\mathrm{EtOH}$ & 0.650 & 0.375 \\
\hline \multirow{3}{*}{ P. aduncum } & $\mathrm{H}_{2} \mathrm{O}+\mathrm{EtOH}$ 50:50 & 58.677 & 10.782 \\
\hline & $\mathrm{MetOH}$ & 168.741 & 17.120 \\
\hline & $\mathrm{EtOH}$ & 2.962 & 0.202 \\
\hline \multirow[t]{3}{*}{ P. amalago } & $\mathrm{H}_{2} \mathrm{O}+\mathrm{EtOH}(50: 50)$ & 10.979 & 1.584 \\
\hline & $\mathrm{MetOH}$ & 4.161 & 0.456 \\
\hline & $\mathrm{EtOH}$ & 0.836 & 0.129 \\
\hline \multirow[t]{3}{*}{ P. marginatum } & $\mathrm{H}_{2} \mathrm{O}+\mathrm{EtOH}(50: 50)$ & 2.214 & 0.319 \\
\hline & $\mathrm{MetOH}$ & $\mathrm{s} / \mathrm{a}$ & $\mathrm{N} / \mathrm{D}$ \\
\hline & $\mathrm{EtOH}$ & 1.389 & 0.165 \\
\hline \multirow[t]{2}{*}{ P. umbellatum } & $\mathrm{H}_{2} \mathrm{O}+\mathrm{EtOH}(50: 50)$ & 3.101 & 0.449 \\
\hline & $\mathrm{MetOH}$ & 4.630 & 1.545 \\
\hline
\end{tabular}

s/a: sin actividad, N/D: no determinada. EtOH-Etanol, MetOH-Metanol, EtOH:H ${ }_{2} \mathrm{O}:$ Hidroalcohólico al $70 \%$.

tivos que lo reportado para extractos de $P$. nigrum y $P$. betle en Schistosoma mansoni y Meloidogyne incognita (Atjanasuppat et al. 2009, Wiratno et al. 2009, Dammini-Premachandra et al. 2014). El extracto etanólico de $P$. aduncum fue el de mayor actividad contra $C$. elegans con una $\mathrm{CL}_{50}$ estimada en 0.650 $\mathrm{mg} \mathrm{mL}^{-1}$, similar a la reportada contra Haemonchus contortus y Melodoigyne incognita, además de tener efecto supresor en la formación de nódulos de plántulas de tomate infestadas con Melodoigyne sp. (Singh y Khuma 2006, Oliveira et al. 2014).

En contraste, se evidenció que el extracto 
Tabla 2. Análisis fitoquímico preliminar de las cuatro especies de Piper.

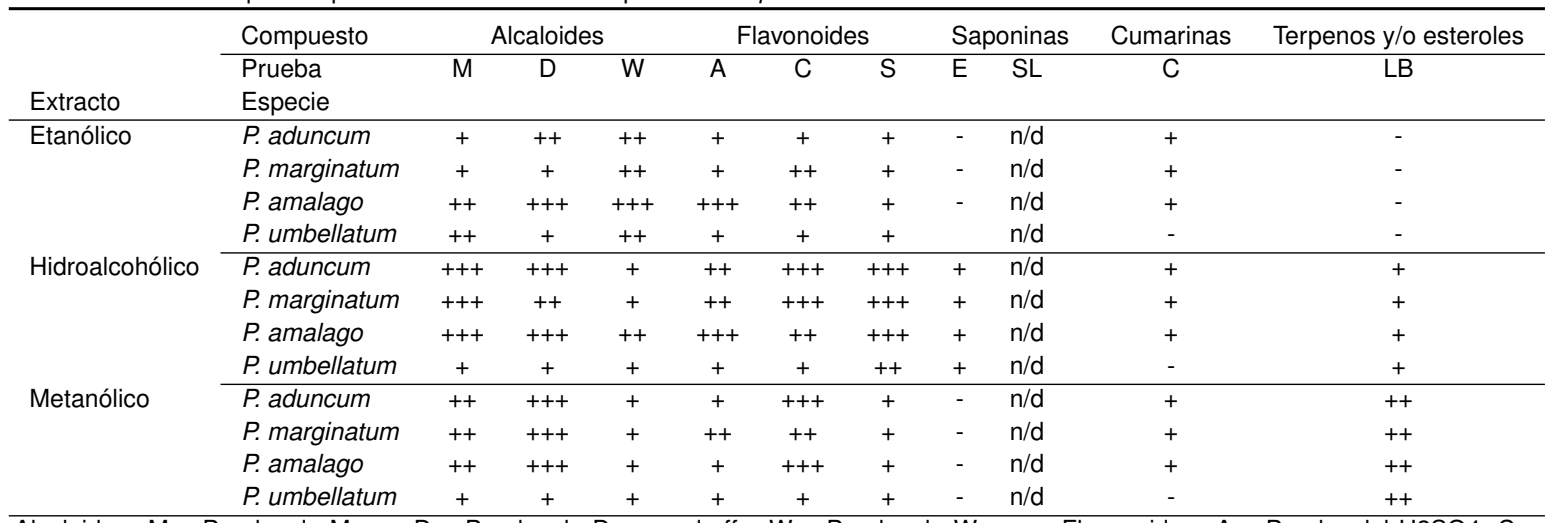

Alcaloides: $\mathrm{M}=$ Prueba de Mayer, $\mathrm{D}=$ Prueba de Drangendorff y $\mathrm{W}=$ Prueba de Wagner. Flavonoides: $\mathrm{A}=\mathrm{Prueba}$ del $\mathrm{H} 2 \mathrm{SO}$, $\mathrm{C}=$ Prueba del $\mathrm{FeCL}_{2}, \mathrm{~S}=$ Prueba de Shinoda. Saponinas: $\mathrm{E}=$ Prueba de la espuma, $\mathrm{SL}=$ Prueba de Lieberman. Cumarinas: $\mathrm{C}=\mathrm{Prueba}$ de fluorescencia. Triterpenos y/o Esteroles: LB = Prueba de Lieberman-Bouchard. Intensidad: mayor +++, media++, baja +, nula - y n/d no determinada.

metanólico de $P$. marginatum no presentó actividad en dosis de $500 \mathrm{mg} \mathrm{mL}^{-1}$, lo que coincide con lo reportado de que el extracto acuoso de esta especie no es efectivo contra Melodoigyne incognita (Vinueza et al. 2006).

Los extractos etanólicos en este estudio presentaron efecto biocida mayor que el indicado en otros estudios, en los cuales los plaguicidas sintéticos como el clorpirifo, carbosulfan y deltramina, tuvieron $\mathrm{CL}_{50}$ estimadas en $19.4,25.3$ y $40 \mathrm{mg} \mathrm{mL}^{-1}$, respectivamente (Wiratno et al. 2009, Sant'anna et al. 2013). Esta función se puede atribuir a los diferentes compuestos secundarios presentes en los extractos, como terpenos y/o esteroles, ácidos grasos, flavonoides, compuestos fenólicos y alcaloidesamidas, las cuales actúan a nivel de sistema nervioso central en insectos y nemátodos (Scott et al. 2008, Suzuki y Yamato 2018). Compuestos como el dilapiol son agentes clastogénicos cuyo isómero apiol se encontró presente en el extracto etanólico de $P$. maginatum, lo que puede explicar su efecto tóxico en $C$. elegans (Razzaghi-Abyaneh et al. 2007, Rafael et al. 2008). Además de taninos, saponinas, glucósidos cianogénicos y otros compuestos reportados en extractos crudos de Piper que pudieran ser causantes de este efecto (Ejele et al. 2012, Varsha y Sonali 2014, Kumar y Babu 2014, Shailesh 2015).

Los perfiles fitoquímicos determinados por HPTLC muestran que existe una diversidad de metabolitos secundarios presentes. Se encontró similitud en los valores de Rf, con una banda de Rf $=0.8$, reportada anteriormente para $P$. nigrum, la cual corresponde a un flavonoide; esto puede indicar que este compuesto es químicamente similar, es decir un homólogo o isómero del mismo (Valencia 1995). Las bandas de alcaloides o alcamidas fueron escasas, y se encontraron en los extractos de tres especies de las cuatro en estudio, el compuesto más común fue el correspondiente al $\mathrm{Rf}=0.6$, que puede corresponder de manera similar a lo reportado por Rajopadhye et al. (2011), para una alcamida presente en P. nigrum (pimenta negra), P. nigrum (pimienta blanca), $P$. longum, $P$. retrofractum, $P$. cubeba y $P$. betle.

Los compuestos mayoritarios en los extractos etanólicos fueron el ácido palmítico y el fitol, ya reportados también para $P$. umbellatum, $P$. betle, P.aduncum, P. auritum, P. decurrens y P. methysticum (Parmar et al. 1997, Lee et al. 2004, Hezekiah et al. 2015). El cadineno fue identificado en sus formas $\beta$ y $\delta$, el cual fue reportado para $P$. marginatum, $P$. amalago y $P$. aduncum (Ferraz et al. 2010, Sant'anna et al. 2015) en el caso de $\beta$ cadineno se ha reportado para P. nigrum (Bagheri et al. 2014).

Los monoterpenos, sesquiterpenos y compuestos fenólicos identificados como $\alpha$-gurjuneno, aromandendreno, aloaromadendreno, L-calameneno, cadaleno, pineno, $\beta$-cubebeno, germacreno $D$, piperotona, cariofileno, piperonal, $\alpha$-cadinol, $\beta$ - 
Tabla 3. Valores de Rf de los perfiles fitoquímicos de los extractos foliares de las cuatro especies de Piper por HPTLC.

\begin{tabular}{|c|c|c|c|c|c|c|}
\hline Extracto & $\begin{array}{l}\text { Compuesto } \\
\text { Fase móvil } \\
\text { Revelador } \\
\text { Especie }\end{array}$ & $\begin{array}{l}\text { Alcaloides } \\
\text { A } \\
\text { D }\end{array}$ & $\begin{array}{l}\text { Flavonoides } \\
\text { F } \\
\text { PN }\end{array}$ & $\begin{array}{l}\text { Cumarinas } \\
\text { C } \\
\text { HS }\end{array}$ & $\begin{array}{l}\text { Saponinas } \\
\text { S } \\
\text { AA }\end{array}$ & $\begin{array}{l}\text { Terpenos } \\
\text { T } \\
\text { VS }\end{array}$ \\
\hline \multirow[t]{4}{*}{ Etanólico } & P. aduncum & & 0.874 & $\begin{array}{l}0.7,0.781 \\
0.886\end{array}$ & $\begin{array}{l}0.057,0.17,0.182,0.287 \\
0.329,0.44,0.512,0.514 \\
0.681,0.746,0.788,0.795 \\
0.863,0.969\end{array}$ & $\begin{array}{l}0.04,0.079,0.119,0.148 \\
0.184,0.223,0.268,0.302 \\
0.707,0.747,0.93\end{array}$ \\
\hline & P. marginatum & $0.572,0.649$ & $0.541,0.813$ & $0.656,0.901$ & $\begin{array}{l}0.262,0.37,0.558,0.614 \\
0.805,0.951\end{array}$ & $\begin{array}{l}0.025,0.096,0.106,0.179, \\
0.241,0.267,0.292,0.429, \\
0.485,0.559,0.697,0.774\end{array}$ \\
\hline & P. amalago & $\begin{array}{ll}0.52, & 0.588 \\
0.646, & 0.786\end{array}$ & 0.752 & $\begin{array}{l}0.648,0.714 \\
0.768\end{array}$ & $\begin{array}{l}0.170,0.284,0.239,0.444 \\
0.478,0.666\end{array}$ & $\begin{array}{l}0.023,0.099,0.123,0.17 \\
0.197,0.265,0.487,0.692\end{array}$ \\
\hline & P. umbellatum & $0.588,0.648$ & 0.739 & $\begin{array}{l}0.646,0.719 \\
0.869\end{array}$ & $0.162,0.390,0.685,0.694$ & $\begin{array}{l}0.018,0.58,0.104,0.126, \\
0.179,0.234,0.250,0.284, \\
0.703,0.883\end{array}$ \\
\hline \multirow[t]{4}{*}{ Hidroalcohólico } & P. aduncum & & 0.885 & $\begin{array}{l}0.793,0.802 \\
0878\end{array}$ & $\begin{array}{l}0.514,0.681,0.795,0.864 \\
0.961\end{array}$ & $\begin{array}{l}0.084,0.111,0.262,0.299, \\
0.692\end{array}$ \\
\hline & P. marginatum & $0.595,0.648$ & 0.693 & $\begin{array}{l}0.487,0.648 \\
0.759\end{array}$ & $\begin{array}{l}0.116,0.167,0.649,0.805 \\
0.869,0.954\end{array}$ & $0.094,0.173,0.212,0.256$ \\
\hline & P. amalago & $\begin{array}{l}0.593,0.632 \\
0.744,0.824\end{array}$ & $\begin{array}{l}0.752,0.876 \\
0.886\end{array}$ & $\begin{array}{l}0.648,0.722 \\
0.769,0.837 \\
0.847,0.898 \\
0.937\end{array}$ & $\begin{array}{l}0.165,0.284,0.319,0.377 \\
0.44,0.67,0.791,0.979\end{array}$ & $\begin{array}{l}0.019,0.097,0.123,0.180 \\
0.199,0.2580 .267,0.484, \\
0.561,0.700\end{array}$ \\
\hline & P. umbellatum & $0.588,0.642$ & & $\begin{array}{l}0.646,0.712 \\
0.761,0.863\end{array}$ & $0.397,0.686,0.786,0.967$ & $\begin{array}{l}0.019,0.052,0.101,0.179 \\
0.14,0.253,0.273,0.297\end{array}$ \\
\hline \multirow[t]{4}{*}{ Metanólico } & P. aduncum & & 0.864 & $0.769,0.878$ & $\begin{array}{l}0.180,0.284,0.321,0.448 \\
0.519,0.68,0.802,0.864 \\
0.973\end{array}$ & $\begin{array}{l}0.132,0.18,0.234,0.267, \\
0.695\end{array}$ \\
\hline & P. marginatum & 0.638 & 0.898 & $\begin{array}{l}0.661,0.742 \\
0.797,0.907\end{array}$ & $\begin{array}{l}0.123,0.173,0,175,0.346 \\
0.626,0.957\end{array}$ & $\begin{array}{l}0.052,0.096,0.177,0.218 \\
0.712\end{array}$ \\
\hline & P. amalago & $0.558,0.637$ & $0.747,0.886$ & $\begin{array}{l}0.653,0.727 \\
0.768\end{array}$ & $\begin{array}{l}0.157,0.289,0.317,0.383, \\
0.411,0.485\end{array}$ & $\begin{array}{l}0.019,0.075,0.114,0.117, \\
0.197,0.256,0.417,0.482, \\
0.697\end{array}$ \\
\hline & P. umbellatum & $\begin{array}{l}0.597,0.592 \\
0.646,0.648\end{array}$ & 0.900 & $\begin{array}{l}0.646,0.761 \\
0.866\end{array}$ & $0.390,0.688$ & $\begin{array}{l}0.023,0.060,0.104,0.179, \\
0.214,0.272,0.702\end{array}$ \\
\hline
\end{tabular}

A: tolueno-cloroformo-etanol (28.5:57:41.5, v/v/v), F: acetato de etilo-ácido fórmico-ácido acético glacial-agua (100:11:11:26, v/v/v/v), C: acetato de etilo-tolueno (1:1 v/v) 10\% ácido acético, S: cloroformo-ácido acético-metanol-agua (60:32:12:8 v/v/v/v), T: tolueno-acetato de etilo (93:7 v/v); D: Dragendorff, PNP: productos naturales polietilenglicol, HS: hidróxido de sodio $10 \%$ en etanol, AA; anisaldehído acético (115 $\mathrm{C})$, VS: vainillina sulfúrica $\left(115^{\circ} \mathrm{C}\right)$.

copaeno, $\alpha$-selineno y pirano estuvieron presentes en los extractos etanólicos analizados. Estos ya se han reportado para las especies de $P$. aduncum, $P$. maginatum, $P$. amalago y $P$. umbellatum (Ferraz et al. 2010, Souto et al. 2012, Oliveira et al. 2014, Brazão et al. 2104, Hezekiah et al. 2015). Por lo anterior se recomienda hacer estudios biodirigidos sobre los compuestos que pudieran ejercer el efecto tóxico o determinar si existe una asociación sinérgica entre los compuestos presentes.

\section{CONCLUSIONES}

Los extractos etanólicos estudiados tienen efecto nematicida sobre $C$. elegans a bajas concentraciones, e inclusive superior a lo reportado en la bibliografía para plaguicidas sintéticos comerciales. Los extractos de Piper amalago mostraron efecto tóxico sobre Caenorhabditis elegans por debajo de $10 \mathrm{mg} \mathrm{mL}^{-1}$. Para las cuatro especies de Piper, los extractos metanólicos fueron los de menor actividad, seguidos de los hidroalcohólicos. El extracto metanólico de $P$. marginatum no tuvo actividad nematicida en las concentraciones evaluadas. Se determinó la presencia de alcaloides, terpenos, esteroles, flavonoides, saponinas y cumarinas de acuerdo con las pruebas fitoquímicas preliminares y los perfiles fitoquímicos por HTPLC. Por medio de la GC-EIMS 
Tabla 4. Composición química del extracto etanólico de Piper marginatum determinado por GC-EIMS.

\begin{tabular}{llccc}
\hline Pico & Compuesto & Tiempo de Retención & Área (\%) & Similitud \\
\hline 11 & (Z) -Muurola-3,5-dieno & 24.892 & 0.34 & 94 \\
13 & Ácido cinámico & 25.935 & 0.48 & 93 \\
14 & $\alpha$-Gurjuneno & 26.605 & 0.67 & 99 \\
15 & Aloaromadendreno & 27.337 & 0.66 & 91 \\
17 & Aromandendreno & 27.849 & 0.3 & 95 \\
18 & $\alpha$-Muurolene & 28.086 & 0.58 & 98 \\
19 & $\beta$-Cubebeno & 28.288 & 0.25 & 95 \\
21 & Biciclogermacreno & 28.678 & 2.8 & 93 \\
23 & L-Calameneno & 29.068 & 0.81 & 90 \\
24 & $\delta$-Cadineno & 29.159 & 0.54 & 98 \\
25 & $\alpha$-Calacoreno & 29.549 & 0.27 & 96 \\
30 & $\beta$-Celineno & 30.713 & 0.56 & 96 \\
31 & Apiol & 30.963 & 6.9 & 90 \\
34 & $\alpha$-Cadinol & 32.128 & 0.74 & 93 \\
35 & Cadaleno & 32.487 & 0.2 & 98 \\
41 & Pineno & 36.157 & 0.93 & 93 \\
44 & Ácido palmítico & 38.339 & 11.14 & 99 \\
46 & Nerolidol & 39.643 & 0.41 & 91 \\
48 & Ácido linoleico & 41.496 & 10.78 & 99 \\
49 & $\alpha$-Ácido linoleico & 41.569 & 6.85 & 99 \\
50 & Ácido esteárico & 41.966 & 3.31 & 99 \\
52 & Dipropil sulfito & 43.489 & 0.69 & 94 \\
59 & Eicosano & 51.462 & 0.66 & 94 \\
52 & $\alpha$-Tocoferol & 52.266 & 0.85 & 94 \\
65 & Campesterol & 57.13 & 0.83 & 91 \\
\hline Similitud: con el espectro reportado con & & & 96 \\
& & & &
\end{tabular}

Tabla 5. Composición química del extracto etanólico de Piper umbellatum determinado por GC-EIMS.

\begin{tabular}{lcccc}
\hline Pico & Compuesto & Tiempo de Retención & Área (\%) & Similitud \\
\hline 1 & Pyranono & 18.078 & 1.98 & 90 \\
8 & $\alpha$-Gurjuneno & 26.709 & 0.82 & 99 \\
9 & Aromandendreno & 27.452 & 0.71 & 93 \\
11 & $\beta$-Cadineno & 28.202 & 0.65 & 95 \\
13 & B-Copaeno & 28.787 & 3.06 & 90 \\
14 & Calameneno & 29.122 & 0.51 & 92 \\
15 & $\delta$-Cadineno & 29.183 & 0.81 & 90 \\
16 & $\alpha$-Selineno & 29.275 & 0.6 & 93 \\
22 & 2,5-dimetil-3-metilen-hepta-1,-dieno & 31.305 & 1.24 & 95 \\
23 & 2-isopropil-5-metil-9-metilen-biciclo-1-deceno (4.4.0) & 31.951 & 0.45 & 90 \\
24 & Cadaleno & 32.255 & 0.85 & 98 \\
25 & Ácido palmítico & 32.609 & 0.2 & 96 \\
31 & Ácido palmítico, éster metil & 38.485 & 12.2 & 99 \\
32 & Fitol & 39.064 & 0.29 & 96 \\
34 & Ácido linoleico & 41.478 & 10.39 & 98 \\
35 & $\alpha$-Ácido linoleico & 41.654 & 11.57 & 99 \\
36 & Ácido esteárico & 41.734 & 7.71 & 99 \\
37 & 42.160 & 3.17 & 99 \\
\hline
\end{tabular}

Similitud: con el espectro reportado con máxima similitud en la base de datos NIST.

se confirmó que cuentan con una diversidad de compuestos en su mayoría de origen terpénico y fenólico, así como componentes de aceites esenciales.

\section{AGRADECIMIENTOS}

El autor del trabajo agrace al CONACYT por la beca 408116 otorgada para la realización de estudios de posgrado. Así mismo al Dr. Rafael Montiel y la Dra. Hilda Ramos del Evolution of Metabolic Diversity-LANGEBIO-CINVESTAV-IPN, Irapuato por la donación de las Cepas N2 y OP50 de C. elegans y E. coli. 
Tabla 6. Composición química del extracto etanólico de Piper amalago determinado por GC-EIMS

\begin{tabular}{llccc}
\hline Pico & Compuesto & Tiempo de Retención & Área (\%) & Similitud \\
\hline 7 & Pirano & 18.066 & 1.38 & 93 \\
8 & Coumaran & 20.278 & 1.21 & 93 \\
9 & Piperonal & 23.417 & 0.47 & 91 \\
21 & Ácido palmítico & 38.381 & 2.74 & 99 \\
22 & Ácido palmítico, éster metil & 39.058 & 0.23 & 90 \\
27 & Fitol & 41.502 & 18.77 & 96 \\
28 & Ácido linoleico & 41.661 & 5.14 & 99 \\
31 & $\alpha$-Ácido linoleico & 42.191 & 0.39 & 95 \\
\hline \multicolumn{2}{l}{ Similitud: } & con el espectro reportado con máxima similitud en la base de datos NIST.
\end{tabular}

Tabla 7. Composición química del extracto etanólico de Piper aduncum determinado por GC-EIMS

\begin{tabular}{llccc}
\hline Pico & Compuesto & Tiempo de Retención & Área (\%) & Similitud \\
\hline 6 & Piperotona & 21.583 & 0.56 & 96 \\
9 & Germacreno D & 25.776 & 0.06 & 90 \\
10 & (Z)-ácido cinámico & 26.050 & 0.13 & 95 \\
11 & Cariofileno & 26.934 & 0.2 & 94 \\
12 & Humuleno & 27.775 & 0.21 & 91 \\
18 & -adineno & 29.275 & 0.19 & 95 \\
40 & Ácido mirístico & 34.236 & 0.08 & 98 \\
52 & Ácido palmítico & 38.503 & 3.07 & 99 \\
53 & Ácido palmítico, éster metil & 39.064 & 0.08 & 95 \\
55 & Fitol & 41.514 & 3.14 & 99 \\
57 & Ácido linoleico & 41.788 & 1.53 & 98 \\
58 & $\alpha$-Ácido linoleico & 42.130 & 0.21 & 94 \\
59 & Ácido esteárico & 42.192 & 0.78 & 99 \\
69 & Estigmasterol & 47.433 & 0.03 & 95 \\
73 & $\gamma$-Sitosterol & 51.151 & 0.43 & 91 \\
\hline Similitud: es el espectro reportado con máxima similitud en la base de datos NIST.
\end{tabular}

\section{LITERATURA CITADA}

Abd-Elgawad, MM, Askary TH (2015) Impact of phytonematodes on agriculture economy. In Askary TH, Martinello PRP (Ed) Biocontrol agents of phytonematodes. CAB International. Wallingford, UK. pp. 3-49.

Agyare C, Spiegler V, Sarkodie H, Asase A, Liebau E, Hensel A (2014) An ethnopharmacological survey and in vitro confirmation of the ethnopharmacological use of medicinal plants as anthelmintic remedies in the Ashanti region, in the central part of Ghana. Journal of Ethnopharmacology 158: 255-263.

Annegowda HV, Tan PY, Mordi MN, Ramanathan S, Hamdan MR, Sulaiman MH, Mansor SM (2013) TLCBioautography-guided isolation, HPTLC and GC-MS-assisted analysis of bioactives of Piper betle leaf extract obtained from various extraction techniques: in vitro evaluation of phenolic content, antioxidant and antimicrobial activities. Food Analytical Methods 6: 715-726.

Atjanasuppat K, Wongkham W, Meepowpan P, Kittakoop P, Sobhon P, Bartlett A, Whitfield PJ (2009) In vitro screening for anthelmintic and antitumor activity of ethnomedicinal plants from Thailand. Journal of Ethnopharmacology 123: 475-482.

Bagheri H, Manap MBA, Solati Z (2014) Antioxidant activity of Piper nigrum L. essential oil extracted by supercritical $\mathrm{CO}_{2}$ extraction and hydro-distillation. Talanta 121: 220-228.

Brazao MA, Brazao F, Maia JG, Monteiro M (2014) Antibacterial activity of the Piper aduncum oil and dillapiole, its main constituent, against multidrug-resistant strains. Boletín Latinoamericano y del Caribe de Plantas Medicinales y Aromáticas, 13: 517-526. 
Carmona-Hernández O, Fernández MS, Palmeros-Sánchez B, Lozada-García JA (2015) Actividad insecticida de extractos etanólicos foliares de nueve piperáceas (Piper spp.) en Drosophila melanogaster. Revista Internacional de Contaminación Ambiental 30: 67-73.

Carmona-Hernández O, Lozada-García JA, Martínez-Hernández MJ, Fernández MS, Torres-Pelayo VR (2016) Piper L. genus potential as natural biocide. Wulfenia Journal 23: 65-95.

Dammini-Premachandra WTS, Mampitiyarachchi H, Ebssa L (2014) Nemato-toxic potential of Betel (Piper betle L.) (Piperaceae) leaf. Crop Protection 65: 1-5.

Del-Prado-Vera IC, Franco-Navarro F, Godinez-Vidal D (2018) Plant parasitic nematodes and management strategies of major crops in Mexico. In: Subbotin S, Chitambar J (eds) Plant parasitic nematodes in sustainable agriculture of North America. Sustainability in plant and crop protection. Springer Cham, Switzerland. pp: 211-239

Domínguez AX (1979) Métodos de investigación fitoquímica. Editorial Limusa, México. 281p.

Ejele AE, Duru IA, Ogukwe CE, Iwu IC (2012) Phytochemistry and antimicrobial potential of basic metabolites of Piper umbellatum, Piper guineense, Ocimum gratissimium and Newbouldia laevis extracts. Journal of Emerging Trends in Engineering and Applied Sciences 3: 09-14.

Ferraz A, Balbino JM, Zini CA, Ribeiro VLS, Bordignon SAL, Von Poser G (2010) Acaricidal activity and chemical composition of the essential oil from three Piper species. Parasitology Research 107: 243-248.

Finney DL (1987) Statistical method in biological assay: The biomathematics of disease. 3th Edition. Oxford University Press. London UK. 522p.

Hezekiah AS, Labunmi L, Joseph OB (2015) Chemical composition and bioactivity of essential oil from Piper umbellatum against grain storage insects. Canadian Journal of Pure and Applied Sciences 9: 3621-3627.

Jaramillo MA, Callejas R, Davidson C, Smith JF, Stevens AC Tepe EJ (2008) A phylogeny of the tropical genus Piper using ITS and the chloroplast intron psbj-petA. Systematic Botany 33: 647-660.

Jensen HR, Scott IM, Sims S, Trudeau VL, Arnason JT (2006) Gene expression profiles of Drosophila melanogaster exposed to an insecticidal extract of Piper nigrum. Journal of Agricultural and Food Chemistry 54: 12891295.

Katiki LM, Ferreira JFS, Zajac AM, Masler C, Lindsay DS, Chagas ACS, Amarante AFT (2011) Caenorhabditis elegans as a model to screen plant extracts and compounds as natural anthelmintic for veterinary use. Veterinary Parasitology 182: 264-268.

Lebot V, Do TKT, Legendre L (2014) Detection of flavokavins (A, B, C) in cultivars of kava (Piper methysticum) using high performance thin layer chromatography (HPTLC). Food Chemistry 151: 554-560.

Lee A, Richars J, Dodson CD (2004) Insolation, synthesis and evolutionary ecology of Piper Amides. In: Dyer LA, Palmer AD (ed). Piper: A model genus for studies of phytochemistry, ecology and evolution. Kluwer Academic-Plenum Publishers, New York USA. pp: 117-139.

Moreno ME, González S, Acevedo L, Morales G, Betancur M, López JJ, Peláez CA (2000) Drosophila melanogaster (Diptera: Drosophilidae): modelo biológico para la estandarización de extractos naturales con actividad insecticida (El Neem- Azadirachta indica- un caso particular). Revista Colombiana de Entomología 62: 51-55.

Oliveira GL, Vieira TM, Nunes VF, Ruas MdeO, Duarte ER, Moreira DdeL, Coelho KMAC Kaplan M, Martins ER (2014) Chemical composition and efficacy in the egg-hatching inhibition of essential oil of Piper aduncum against Haemonchus contortus from sheep. Brazilian Journal of Pharmacognosy 24: 288-292. 
Ow MC, Hall SE (2015) A method for obtaining large populations of synchronized Caenorhabditis elegans dauer larvae. In: Strange K (ed) C. elegans: Methods and applications. Humana Press. New Jersey, USA. pp: 209-219.

Parmar VS, Jain SC, Bisht KS, Jain R, Taneja P, Jha A, Om D, Tyagi AK, Prasad J, Wengel TCE, Olsen, Boll PM (1997) Phytochemistry of the genus Piper. Phytochemistry 46: 591-673.

Pehlivan HD, Kaşkavalci G, Uludamar EBK, Toktay, H, Elekcioğlu H (2020) Identification and prevalence of potato cyst nematodes and root-knot nematodes in the potato production areas of İzmir Province, Turkey. Turkish Journal of Entomology 44: 259-272.

Porta de la Riva M, Fontrodona L, Villanueva A, Cerón J (2012) Basic Caenorhabditis elegans methods: synchronization and observation. Journal of Visualized Experiments 64: 4019-4019.

Rafael MS, Hereira-Rojas WJ, Roper J, Nunomura SM, Tadei WP (2008) Potential control of Aedes aegypti (Diptera: Culicidae) with Piper aduncum L. (Piperaceae) extracts demonstrated by chromosomal biomarkers and toxic effects on interphase nuclei. Genetics and Molecular Research 7: 772-781.

Rajopadhye AA, Namjoshi TP, Upadhye AS (2012) Rapid validated HPTLC method for estimation of piperine and piperlongumine in root of Piper longum extract and its commercial formulation. Brazilian Journal of Pharmacognosy 22: 1355-1361.

Razzaghi-Abyaneh M, Yoshinari T, Shams-Ghahfarokhi M, Rezaee MB, Nagasawa H, Sakuda S (2007) Dillapiol and Apiol as specific inhibitors of the biosynthesis of aflatoxin $\mathrm{G} 1$ in Aspergillus parasiticus. Bioscience, Biotechnology, and Biochemistry 71: 2329-2332.

Riffault L, Destandau E, Pasquier L, André P, Elfakir C (2014) Phytochemical analysis of Rosa hybrida cv. "Jardin de Granville" by HPTLC, HPLC-DAD and HPLC-ESI-HRMS: Polyphenolic fingerprints of six plant organs. Phytochemistry 99: 127-134.

Robles-Zepeda RE, Molina-Torres J, Lozoya-Gloria E, López MG (2006) Volatile organic compounds of leaves and flowers of Montanoa tomentosa. Flavour and Fragrance Journal 21: 225-227.

Roeber F, Jex AR, Gasser RB (2013) Impact of gastrointestinal parasitic nematodes of sheep, and the role of advanced molecular tools for exploring epidemiology and drug resistance - an Australian perspective. Parasites y Vectors 6: 1-13.

Roh JY, Choi J (2008) Ecotoxicological evaluation of chlorpyrifos exposure on the nematode Caenorhabditis elegans. Ecotoxicology and Environmental Safety 71: 483-489.

Sant'anna V, Vommaro RC, de Souza W (2013) Caenorhabditis elegans as a model for the screening of anthelminthic compounds: Ultrastructural study of the effects of albendazole. Experimental Parasitology 135: $1-8$.

Scott IM, Jensen HR, Philogène BJR, Arnason JT (2008) A review of Piper spp. (Piperaceae) phytochemistry, insecticidal activity and mode of action. Phytochemistry Reviews 7: 65-75.

Shailesh (2015) Preliminary phytochemical and antimicrobial screening of Syzygium aromaticum, Elettaria cardamomum and Piper nigrum extracts. Journal of Pharmacognosy and Phytochemistry 4: 85-89.

Singh SK, Khurma UR (2006) Assessing the potential of kava (Piper methysticum Forst) and wild kava (Piper aduncum L.) as organic amendments for managing root-knot nematodes. South Pacific Journal of Natural Science 26: 33-38.

Soberon GV, Rojas C, Saavedra J, Kato MJ, Delgado GE (2006) Acción biocida de plantas de Piper tuberculatum Jacq. sobre Diatrea saccharalis (Lepidóptera, Pyralidae). Revista Peruana de Biología 13: 107-112. 
Souto RNP, Harada AY, Andrade EHA, Maia JGS (2012) Insecticidal activity of Piper essential oils from the Amazon against the fire ant Solenopsis saevissima (Smith) (Hymenoptera: Formicidae). Neotropical Entomology 41: 510-517.

Suzuki T, Yamato S (2018) Mode of action of piperovatine, an insecticidal piperamide isolated from Piper piscatorum (Piperaceae), against voltage-gated sodium channels. Neurotoxicology 69: 288-295.

Upadhya V, Pai S, Ankad G, Hegde H (2016) Pharmacognostic screening of Piper trichostachyon fruits and its comparative analysis with Piper nigrum using chromatographic techniques. Pharmacognosy Magazine 12: 152-158.

Valencia OC (1995) Fundamentos de fitoquímica. Trillas. México. 235p.

Varsha H, Sonali S (2016) Studies on qualitative phytochemical analysis of selected species of Piper. International Journal of Life Scicences Special Issue A2: 156-158.

Villaseñor JL (2016) Catálogo de las plantas vasculares nativas de México. Revista Mexicana de Biodiversidad 87: 559-902.

Vinueza PSM, Crozzoli R, Perichi G (2006) Evaluación in vitro de extractos acuosos de plantas para el control del nematodo agallador Meloidogyne incognita. Fitopatología Venezolana19: 26-31.

Wang K, Luo C, Liu H, Xu J, Sun W, Zhou L (2012) Nematicidal activity of the alkaloids from Macleaya cordata against certain nematodes. African Journal of Agricultural Research 7: 5925-5929.

Wiratno TD, Van den Bergb H, Riksend JAG, Rietjensb IMCM, Djiwantia SR, Kammengad JE, Murk AJ (2009) Nematicidal activity of plant extracts against the root-knot nematode, Meloidogyne incognita. The open natural products journal 2: 77-85. 